\title{
Establishment of Beauveria bassiana (Balsamo) Vuillemin as an Endophytein Cotton
}

\author{
M. Amutha* \\ Department of Entomology, ICAR- Central Institute for Cotton Research, \\ Regional Station, Coimbatore - 641 003, Tamil Nadu, India \\ *Corresponding author
}

\begin{tabular}{|c|c|}
\hline & A B S T R A C T \\
\hline & \multirow{6}{*}{$\begin{array}{l}\text { Entomopathogenic fungal bioagent, Beauveria bassiana is a potential target specific and } \\
\text { eco friendly alternative for chemical control. B. bassiana despite being highly pathogenic } \\
\text { to insects in the laboratory condition, it is less efficient in the field condition because, the } \\
\text { external application of spray formulations of B. bassiana adversely get affected by abiotic } \\
\text { factors. To reduce this limitation, endophytes have received considerable attention as a } \\
\text { promising supplement or alternative to chemical control. This work aims to establish } \\
\text { entomopathogen B. bassiana as endophyte into cotton plant system by artificial } \\
\text { inoculation methods. B. bassiana was inoculated into cotton plant by four different } \\
\text { methods viz., seed immersion, seed coating, foliar spray and soil drenching. The recovery } \\
\text { of } B \text {. bassiana and other endophytic fungi was evaluated by culture methods at one month } \\
\text { and two months of post inoculation. Among the different inoculation methods, the foliar } \\
\text { application method recorded with highest colonization percentage. The } B \text {. bassiana was } \\
\text { recovered from cotton plant parts even at two months after inoculation. In addition to } B \text {. } \\
\text { bassiana recovery, the maximum of } 157 \text { numbers of other endophytic fungi were also } \\
\text { obtained at two months post-inoculation, followed by } 144 \text { numbers at one month post } \\
\text { inoculation. The results revealed that, B. bassiana can able to establish as endophyte in } \\
\text { cotton plants. }\end{array}$} \\
\hline & \\
\hline $\begin{array}{l}\text { Cotton, } \\
\text { Entomopathogen, } \\
\text { Beauveria } \\
\text { bassiana, } \\
\text { Endophytes, } \\
\text { Colonization. } \\
\end{array}$ & \\
\hline Article Info & \\
\hline $\begin{array}{l}\text { Accepted: } \\
\text { 29 May } 2017 \\
\text { Available Online: } \\
\text { 10 June } 2017\end{array}$ & \\
\hline & \\
\hline
\end{tabular}

\section{Introduction}

Cotton pest management has always been an immensely challenging task for entomologists. Continuous and indiscriminate use of insecticides for management of insects have increased the selection pressure and leading to resistance to insects. Therefore, alternate options of pest control are much awaited. The need of the hour is development of eco-friendly, microbe based insecticides which act differently from chemicals, thereby providing least chance to develop resistance. Among various microbial bioproducts,
Beauveria bassiana popularly used. So far, prevailing microbial pesticides are being mainly used as foliar application (Ratna Kumari et al., 2014) but its efficacy adversely affected by abiotic factors (Thompson et al., 2006).

Endophytes have received increasing attention as a promising alternative to chemical control. Endophytic microorganisms reside asymptomatically within higher plants, inhabiting leaves, stems and roots without any 
apparent harm to the plant (Jalgaonwala et al., 2011). Endophytic fungi are important because they produce secondary metabolites with a range of potential uses in agriculture (Selim et al., 2012). Furthermore, some endophytes protect plants from subsequent attack by insect pests and plant pathogens (Azevedo et al., 2000). B. bassiana also exist as natural endophytes and can be introduced into plants using several artificial plant inoculation methods (Vega, 2008; Brownbridge et al., 2012).

The use of $B$. bassiana as an artificial endophyte in cotton would potentially solve the constraints limiting its field application. Furthermore, once established as an endophyte, $B$. bassiana might offer the most suitable and season long protection against the insect pests of cotton. Hence, in the present investigation, the colonization efficiency of $B$. bassiana on the $B t$ and non- $B t$ cotton by different methods of inoculation with different concentration were compared. This study provides the baseline data for the further detailed studies.

\section{Materials and Methods}

\section{B. bassiana inoculum preparation}

The B. bassiana culture was obtained from National Bureau for Agriculturally Important Insects (NBAII) Bengaluru, Karnataka, India. The B. bassiana strain was subcultured on Sabouraud dextrose agar medium supplemented with yeast extract (SDAY) (10g peptone, $20 \mathrm{~g}$ dextrose, $5 \mathrm{~g}$ yeast extract and

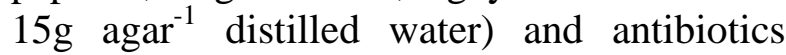
$(0.1 \mathrm{~g}$ penicillin, $0.2 \mathrm{~g}$ streptomycin and $0.05 \mathrm{~g}$ chlortetracycline ${ }^{-1}$ SDAY) in $55 \mathrm{~mm}$ diameter Petri dishes. The Petri dishes containing the B. bassiana were incubated for three weeks in the laboratory. Conidia were harvested by gently scraping them off the surface of the dried medium using a sterile scalpel blade.
Conidial concentration was determined by dissolving $0.1 \mathrm{~g}$ conidial powder in $10 \mathrm{ml}$ sterile deionized water containing $0.01 \%$ Tween 80 in a sterile $500 \mathrm{ml}$ bottle. After vortexing for one minute serial dilutions were made, and the conidial concentration determined using an improved Neubauer haemocytometer. The conidial concentration for each treatment was adjusted to $1 \times 10^{6}$, $1 \times 10^{7}$ and $1 \times 10^{8}$ conidia $\mathrm{ml}^{-1}$. Germination test of conidia was done before inoculating in the plants.

\section{Inoculation methods}

Bunny $B t$ and respective non $B t$ seeds were used for these experiments. B. bassiana was inoculated by four different methods: (1) Seed immersion (2) Seed coating (3) Foliar spray and (4) Soil drenching. Twenty plants were used per inoculation.

For seed immersion treatment, $50 \mathrm{~g}$ of seeds were immersed into $10 \mathrm{ml}$ of $B$. bassiana conidial suspension of various concentrations for 6 hours. After that the inoculated seeds were dried on sterile tissue paper for $30 \mathrm{~min}$ and they were sown in $15 \mathrm{~cm}$ diameter pots. Seeds soaked in sterile distilled water containing $0.01 \%$ Tween 80 were used as control. Seed coating was done by adding $1 \mathrm{~g}$ of $B$. bassiana conidial suspension of various concentrations along with methyl cellulose coated with cotton seeds. For control seeds were coated with methyl cellulose alone. The foliar spray inoculation method was performed with a hand sprayer to inoculate each seedling with $10 \mathrm{ml}$ conidial suspension of various concentrations at fifteen days after emergence of seedlings. The spray was directed mainly to the leaves but also incidentally coated the stems. To avoid conidial runoff to the soil, the soil top of each pot was covered with aluminium foils. For the soil drench inoculation method, $10 \mathrm{ml}$ conidial suspension of various concentrations 
was applied around the root zone of each seedling. In the control, sterile $0.01 \%$ Tween 80 applied in the same way as mentioned above. After inoculation, each plant was covered with a plastic bag for $24 \mathrm{hrs}$ to maintain a high level of humidity. The inoculated plants were kept in room temperature and natural light conditions of 12:12 $\mathrm{h}$ and watered daily.

\section{Evaluation for presence of $B$. bassiana in $B t$ and non-Bt cotton tissues}

The recovery of $B$. bassiana and other endophytic fungi was evaluated by culture methods at one month and two months of post inoculation. Stems were cut off (about $5 \mathrm{~cm}$ above the stem base) from the roots using a sterile blade. The leaves were randomly selected from the middle section of the seedling. Similarly, two parts of the stem were sampled, one towards the middle of the plant and the second one closer to the soil surface. The leaves were cut into $1 \mathrm{~cm}^{2}$ sections, sterilized in a laminar airflow cabinet by dipping in $0.5 \%$ Sodium hypochlorite suspension for two minutes followed by dipping in $75 \%$ ethanol for 2 min. The tissues were dried on sterile paper towels and placed in $55 \mathrm{~mm}$ petri dishes containing SDAY. The medium was supplemented with antibiotics to prevent bacterial contamination. A total of 20 plants and 200 tissue subsamples were evaluated for each treatment during the course of one period of inoculation. The Petri dishes were incubated for four days at $25 \pm 2^{0} \mathrm{C}$, in the laboratory, after which all plant samples were visually examined for fungal outgrowth.

B. bassiana colony was characterized as based on white dense mycelia, becoming cream to pale yellow at the edge (Humber, 1997). Percentage colonization was calculated as number of samples exhibiting $B$. bassiana outgrowth per total number of samples, results are expressed as the percentage of plants positive for the presence of $B$. bassiana after inoculation. The number of fungal endophytes other than $B$. bassiana isolated from plants at one month and two months post-inoculation were also assessed and identified based on morphological character by service provided by Agharkar Institute, Pune, India.

\section{Results and Discussion}

\section{Colonization of $B$. bassiana in non- $B t$ cotton as an endophyte}

All fungal inoculation methods were effective in introducing $B$. bassiana into the plant, although at different levels of efficiency. When the data are combined for all inoculation methods, the total percent of plants that tested positive for B. bassiana i.e. colonisation of $B$. bassiana was $35 \%, 39.5 \%$ and $40 \%$ at concentrations of $10^{6}, 10^{7}$ and $10^{8}$ respectively at one month post inoculation, $42.5 \%, 48.5 \%$ and $68 \%$ at concentrations of $10^{6}, 10^{7}$ and $10^{8}$ respectively at two months post inoculation (Fig. 1).

At two months post inoculation, in foliar application method, highest colonization of $32 \%$ was observed in leaf samples followed by $21 \%$ in stem samples and 8 and $5.5 \%$ in stem and leaf samples respectively in soil drenching method at the concentration of $1 \mathrm{x}$ $10^{8}$ conidia $\mathrm{ml}^{-1}$. Similarly at one month post inoculation, in foliar application method, the highest colonization of 23.5 and $14 \%$ was observed in leaf samples and stem samples respectively at $1 \mathrm{x} \quad 10^{8}$ conidia $\mathrm{ml}^{-1}$ concentration. Whereas, in the same inoculation method the colonization was 21 and $19.5 \%$ at $1 \times 10^{7}$ conidia $\mathrm{ml}^{-1}$ concentrations in leaf and stem samples respectively (Fig. 1). In seed immersion and seed coating methods of inoculation there were no colonization was observed at $10^{6}$ and 
$10^{7}$ concentrations. But at $10^{8}$ concentration, it shows very low percentage of colonization i.e. less than one per cent. B. bassiana was not isolated from any of the samples from control plants.

In soil drenching method of inoculation, per cent colonization of $B$. bassiana was high in stem, than in leaves at both the sampling periods, whereas, in foliar application method the per cent colonization was high in leaf than in stem.

Irrespective of the inoculation methods, the total samples positive for $B$. bassiana colonization was $29 \%$ in leaves at $10^{8}$ concentration followed by $23 \%$ in stem at $10^{7}$ and $20.50 \%$ in stems at $10^{6}$ concentrations at one month post inoculation. Similarly at two months post inoculation it was $39 \%$ at $10^{8}$ in leaf samples followed by $30.50 \%$ in stem, $28.50 \%$ in leaf at $10^{7}$ concentrations (Fig. 1).

In the present study, at two months post inoculation, $B$. bassiana was successfully reisolated from the interior of stem and leaves of cotton plants, clearly indicating that cotton can serve as a suitable host for $B$. bassiana endophyte.

B. bassiana has been established as an endophyte in various plants by different methods of inoculation such as foliar sprays, radical dressing, root and rhizome immersion, seed coating and soil drenching (Parsa et al., 2013).

It is speculated that exposure to high doses may increase colonization and persistence of endophytic B. bassiana in plant tissues. Recovery from stems and leaves also shows that B. bassiana can translocate throughout the plant tissues.

The lack of any visual symptoms on the seedlings also would indicate that $B$. bassiana can colonize this plant without causing detriment to the host.

\section{Colonization of $B$. bassiana in $B t$ cotton as an endophyte}

At two months post inoculation, in foliar application method highest colonization of $30.5 \%$ was observed in leaf samples followed by $19 \%$ in stem samples and 7 and $9 \%$ in stem and leaf samples respectively in soil drenching method at $10^{8}$ concentration. At one month post inoculation, in foliar application method the highest colonization of 20.5 and $12 \%$ was observed in leaf samples and stem samples respectively at $10^{8}$ concentration (Fig. 2).

The colonization of $B$. bassiana were observed in all the concentration tested $(1 \mathrm{x}$ $10^{6}, 10^{7}$ and $10^{8}$ ) both in soil drenching and foliar application methods. In seed immersion and seed coating method there were no colonization was observed at $10^{6}$ and $10^{7}$ concentrations. But at $10^{8}$ it shows very low colonization i.e. not more than two per cent. B. bassiana was not isolated from any of the samples from control plants. In soil drenching method of inoculation at both the sampling periods the per cent colonization was high in stem than in leaves whereas, in foliar application method the per cent colonization was high in leaf than stem (Fig. 2).

The colonisation of B. bassiana in Bt and non Bt cotton as endophyte was revealed that there were no significant differences observed regarding colonization. Similar trend were reported in corn by Lewis et al., (2001).

In the current study, B. bassiana colonization was differed among the plant parts isolated. Many endophytic fungi show a certain degree of tissue specificity because they are adapted to particular conditions present in a given organ. 
Table.1 Endophytic fungi isolated from cotton plants at one and two months post inoculation

\begin{tabular}{|c|c|c|c|c|c|c|c|c|c|c|c|c|c|c|}
\hline \multirow{3}{*}{ Isolates } & \multirow{3}{*}{$\mathbf{n}$} & \multicolumn{6}{|c|}{ One month post inoculation } & \multirow{3}{*}{$\mathbf{n}$} & \multicolumn{6}{|c|}{ Two months post inoculation } \\
\hline & & \multicolumn{2}{|c|}{ Leaf } & \multicolumn{2}{|c|}{ Stem } & \multicolumn{2}{|c|}{ Total } & & \multicolumn{2}{|c|}{ Leaf } & \multicolumn{2}{|c|}{ Stem } & \multicolumn{2}{|c|}{ Total } \\
\hline & & $\mathbf{n}$ & $\%$ & $\mathbf{n}$ & $\%$ & $\mathbf{n}$ & $\%$ & & $\mathbf{n}$ & $\%$ & $\mathbf{n}$ & $\%$ & $\mathbf{n}$ & $\%$ \\
\hline Fusarium solani & 144 & 13 & 9.03 & 8 & 5.56 & 21 & 14.58 & 157 & 16 & 10.19 & 6 & 3.82 & 22 & 14.01 \\
\hline Fusarium oxysporum & 144 & 12 & 8.33 & 6 & 4.17 & 18 & 12.50 & 157 & 14 & 8.92 & 7 & 4.46 & 21 & 13.38 \\
\hline Fusarium sp. & 144 & 12 & 8.33 & 10 & 6.94 & 22 & 15.28 & 157 & 13 & 8.28 & 6 & 3.82 & 19 & 12.10 \\
\hline Penicillium piceum & 144 & 7 & 4.86 & 5 & 3.47 & 12 & 8.33 & 157 & 9 & 5.73 & 5 & 3.18 & 14 & 8.92 \\
\hline Aspergillus flavus & 144 & 11 & 7.64 & 5 & 3.47 & 16 & 11.11 & 157 & 11 & 7.01 & 7 & 4.46 & 18 & 11.46 \\
\hline Aspergillus terrus & 144 & 10 & 6.94 & 4 & 2.78 & 14 & 9.72 & 157 & 9 & 5.73 & 6 & 3.82 & 15 & 9.55 \\
\hline Geotrichum candidum & 144 & 2 & 1.39 & 1 & 0.69 & 3 & 2.08 & 157 & 2 & 1.27 & 0 & 0.00 & 2 & 1.27 \\
\hline Pestalotiopsis uvicola & 144 & 3 & 2.08 & 0 & 0.00 & 3 & 2.08 & 157 & 2 & 1.27 & 1 & 0.64 & 3 & 1.91 \\
\hline Nigrospora sphaerica & 144 & 3 & 2.08 & 1 & 0.69 & 4 & 2.78 & 157 & 2 & 1.27 & 0 & 0.00 & 2 & 1.27 \\
\hline Alternaria sp. & 144 & 4 & 2.78 & 3 & 2.08 & 7 & 4.86 & 157 & 7 & 4.46 & 4 & 2.55 & 11 & 7.01 \\
\hline Chaetomium sp. & 144 & 3 & 2.08 & 0 & 0.00 & 3 & 2.08 & 157 & 2 & 1.27 & 1 & 0.64 & 3 & 1.91 \\
\hline Paecilomyces sp. & 144 & 2 & 1.39 & 0 & 0.00 & 2 & 1.39 & 157 & 2 & 1.27 & 0 & 0.00 & 2 & 1.27 \\
\hline Curvularia sp. & 144 & 2 & 1.39 & 1 & 0.69 & 3 & 2.08 & 157 & 2 & 1.27 & 0 & 0.00 & 2 & 1.27 \\
\hline Cladosporium sp. & 144 & 2 & 1.39 & 0 & 0.00 & 2 & 1.39 & 157 & 2 & 1.27 & 0 & 0.00 & 2 & 1.27 \\
\hline Phomopsis archeri & 144 & 2 & 1.39 & 0 & 0.00 & 2 & 1.39 & 157 & 2 & 1.27 & 1 & 0.64 & 3 & 1.91 \\
\hline Phoma exigua & 144 & 2 & 1.39 & 0 & 0.00 & 2 & 1.39 & 157 & 2 & 1.27 & 0 & 0.00 & 2 & 1.27 \\
\hline Acremonium sp. & 144 & 2 & 1.39 & 0 & 0.00 & 2 & 1.39 & 157 & 1 & 0.64 & 0 & 0.00 & 1 & 0.64 \\
\hline $\begin{array}{l}\text { Non sporulating } \\
\text { hyphae }\end{array}$ & 144 & 6 & 4.17 & 2 & 1.39 & 8 & 5.56 & 157 & 10 & 6.37 & 5 & 3.18 & 15 & 9.55 \\
\hline Total & & 98 & 68.05 & 46 & 31.93 & 144 & 99.99 & & 108 & 68.8 & 49 & 31.2 & 157 & 100 \\
\hline
\end{tabular}

Percentages are based on 144 positive fungal isolations at one month post-inoculation and 157 at two months post-inoculation. 


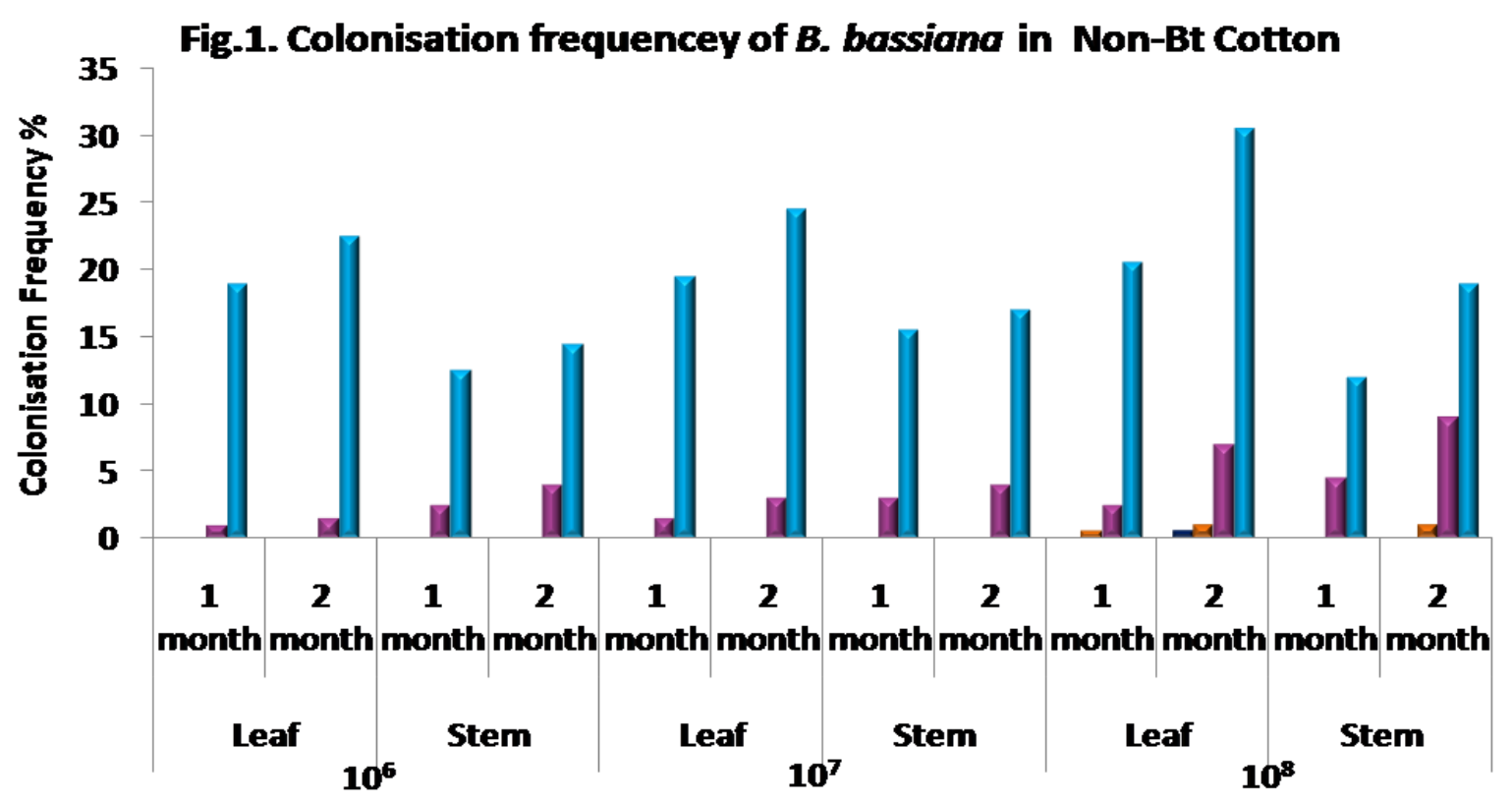

a Seed Immersion $\square$ Seed coating $\square$ Soil drenching $\square$ Foliar application

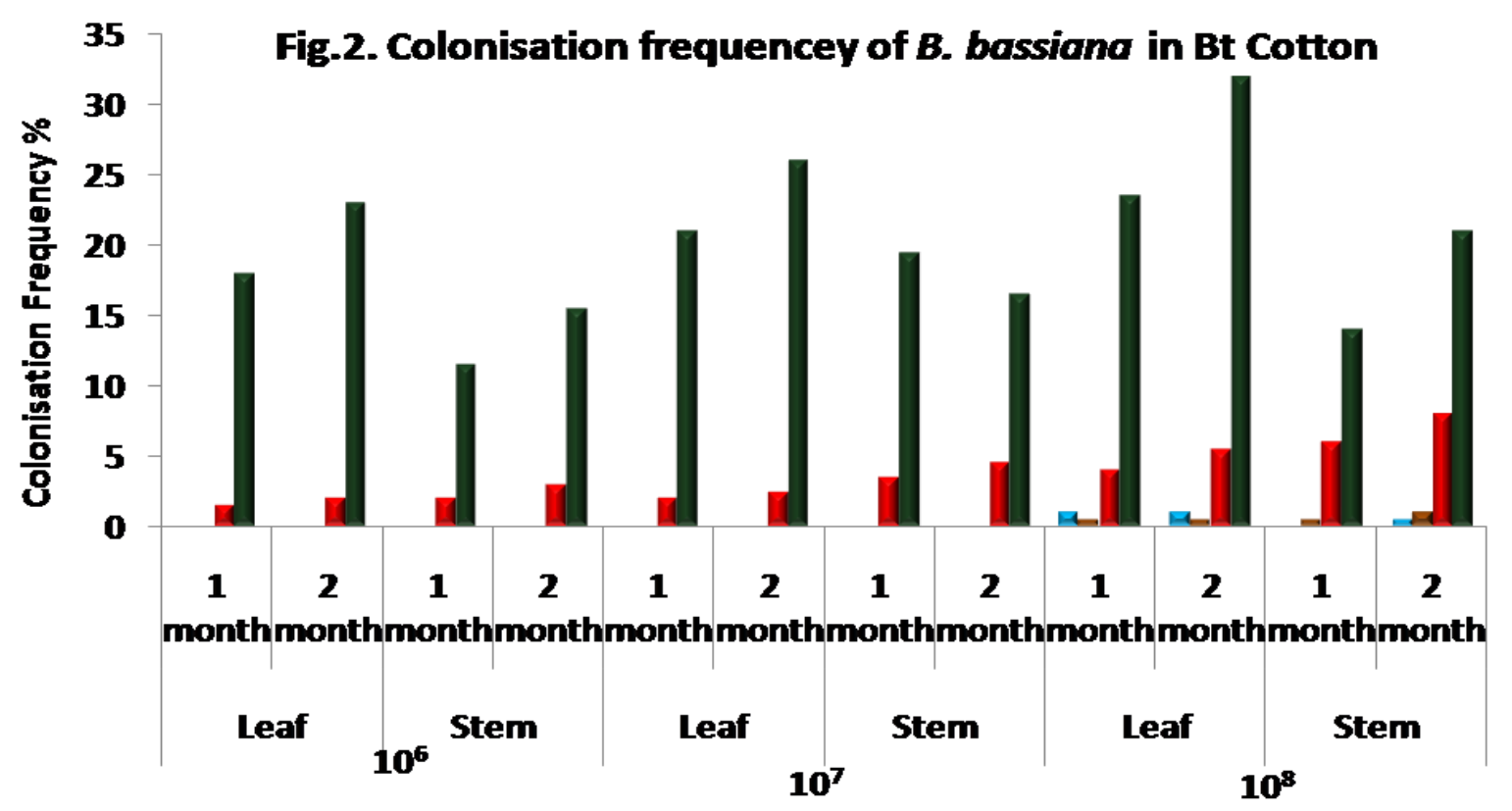

Q Seed Immersion a Seed coating

Soil drenching $\square$ Foliar application

Differential $B$. bassiana colonization on plant parts was demonstrated in corn and cocoa (Posada and Vega, 2005). In corn, the fungus was most frequently isolated from the internode below the primary ear and less frequently from the leaf collar at the primary ear. In cocoa, colonization rates in roots were higher than those in stems and leaves. The reason for the lack of endophytic colonization in seeds treated with $B$. bassiana is not clear and requires further investigation. 
Fungal endophytes isolated from cotton plant

While isolation of B. bassiana from the 200 subsamples, other than B. bassiana, number of fungal endophytes present in the samples were recorded at one and two months post inoculation. At one month post inoculation, 144 isolates were recovered and identified as belonging to 17 species of fungus, one were non-sporulating hyphae whereas, at two month of post inoculation 157 isolates were recovered and belongs to 17 species of fungus (Table 1).

The most frequently isolated fungal species at one and two months post-inoculation were Fusarium, Pencillium piceum, Aspergillus flavus and A. terrus. Similar results were obtained in other endophytic studies also (Rajagopal and Suryanarayanan, 2000). Occurrence of non sporulating hyphae was observed both in leaves and stem samples at both the sampling period (Table 1). Occurrence of sterile mycelium as endophytes is not unusual (Bills, 1996).

At one month post inoculation, the highest number of endophytes recovered from leaves (98) followed by stems (46) similarly, at two months post-inoculation, the highest incidence of endophytes recovered from leaves (108) followed by stems (49) (Table 1). There are many fungi isolates occurred as endophytes in cotton also documented. McGee (2002) found that 13 fungal morpho species, including Alternaria spp., Phomopsis spp., and Fusarium spp. occurred as endophytes in leaves of cotton. Seventeen fungal genera, of which Phoma, Alternaria, Fusarium, Botryo sphaeria, Dichomera and Phomopsis were the common genera, were recovered from the stems of Gossypium. Phoma spp., Fusarium spp., and Phomopsis spp. are common fungal endophytes in both tropical and temperate climates (Schulz, 2005).
In conclusion, the results of this study indicated that the $B$. bassiana can form an endophytic relationship with cotton plants and foliar spray and soil drenching inoculation methods were the best method for the delivery of $B$. bassiana in to the cotton plants. As a result the $B$. bassiana inoculum load most likely remains stable in the cotton plant ever and protect the plant from herbivores. $B$. bassiana becomes an endophyte introduces the possibility that the fungus might naturally recycle in the cotton ecosystem. No significant differences were observed in colonization of $B$. bassiana as endophyte in $B t$ and non- $B t$ cotton plants. The success of artificial inoculation of $B$. bassiana as endophyte into cotton plants determines many future works. It should focus on fine tuning of methodology to optimise the long term establishment of B. bassiana on cotton plant and inoculated plants will be evaluated for its virulence against major pests of cotton.

\section{Acknowledgement}

The author is thankful to the Director and Project co-ordinator and Head of ICARCentral Institute for Cotton Research, for providing necessary facilities during this study.

\section{References}

Azevedo, J.L., Maccheroni, J.W., Pereira, J. and Araujo, W.L. 2000. Endophytic microorganisms: a review on insect control and recent advances on tropical plants. Electronic Journal of Biotechnology, 3, 40-65.

Bills, G.F. 1996. Isolation and analysis of endophytic fungal communities from woody plants. In: Endophytic fungi in grasses and woody plants: Systematics, ecology, and evolution (Redlin, S.C. and Carris, L.M., eds.), APS Press, pp. 31-65. 
Brownbridge, M., Reay, S.D., Nelson, T.L. and Glare, T.R. 2012. Persistence of Beauveria bassiana (Ascomycota: Hypocreales) as an endophyte following inoculation of radiata pine seed and seedlings. Biological Control, 61, 194 200.

Humber, R.A. 1997. Fungi: Identification. In: Manual of Techniques in Insect Pathology (Leacey. ed), Academic Press, Washington, USA, pp. 153-185.

Jalgaonwala, R.E., Mohite, B.V. and Mahajan, R.T. 2011. A review: natural products from plant associated endophytic fungi. Journal of Microbiology and Biotechnology Research, 1, 21-32.

Lewis, L.C., Bruck, D.J., Gunnarson, R.D. and Bidne, K.G. 2001. Assessment of plant pathogenicity of endophytic Beauveria bassiana in Bt transgenic and non transgenic corn. Crop Science, 41, 1395-1400.

McGee, P.A. 2002. Reduced growth and deterrence from feeding of the insect pest Helicoverpa armigera associated with fungal endophytes from cotton. Australian Journal of Experimental Agriculture, 42, 995-999.

Parsa, S., Ortiz, V. and Vega, F.E. 2013. Establishing fungal entomopathogens as endophytes: towards endophytic biological control. Journal of Visualised Experiments, e50360.http:// dx.doi.org/10.3791/50360.

Posada, F. and Vega, F.E. 2005. Establishment of the fungal entomopathogens Beauveria bassiana (Ascomycota: Hypocreales) as an endophyte in cocoa seedlings (Theobroma cacao). Mycologia, 97 (6), 1195-1200.

Rajagopal, K. and Suryanarayanan, T.S. 2000. Isolation of endophytic fungi from the leaves of neem (Azadirachta indica A.Juss). Current Science, 78 (11), 13751378.

Ratnakumari, B., Vijaya Bharathi, R., Srinivas, V. and Gopalakrishnan, S. 2014. Microbes as interesting source of novel insecticides: A review. African Journal of Biotechnology, 13 (26) p.2582-2592

Schulz, B. and Boyle, C. 2005. Fungal endophyte continum. Mycological Research, 109, 661-686.

Selim, K.A., El-Beih, A.A., Abdel-Rahman, T.M. and El-Diwany, A.I. 2012. Biology of endophytic fungi. Current Research in Environmental and Applied Mycology, 2, 31-82.

Thompson, S.R., Brandenburg, R.L. and Arends, J.J. 2006. Impact of moisture and UV degradation on Beauveria bassiana (Balsamo) Vuillemin conidial viability in turfgrass. Biological Control, 39, 401-407.

Vega, F.E., Posada, F., Aime, M.C., PavaRipoll, M., Infante, F. and Rehner, S.A. 2008. Entomopathogenic fungal endophytes. Biological Control, 46, 7282

\section{How to cite this article:}

Amutha, M. 2017. Establishment of Beauveria bassiana (Balsamo) Vuillemin as an Endophyte in Cotton. Int.J.Curr.Microbiol.App.Sci. 6(6): 2506-2513.

doi: https://doi.org/10.20546/ijcmas.2017.606.298 